\title{
Canadian Jewish Heritage Material Repositories / Liste de référence de tous les dépôts du patrimoine juif du Canada
}




\section{ALEX DWORKIN CANADIAN JEWISH ARCHIVES /} ARCHIVES JUIVES CANADIENNES ALEX DWORKIN, Montreal

Suite 211, 4810 Rue Jean-Talon Ouest, Montreal, QC, H4P 2N5

Archives Director: Janice Rosen

Tel: (514) 931-7531 | archives@cjarchives.ca | https://www.cjarchives.ca/en/

ARON MUSEUM, TEMPLE EMANU-EL-BETH-SHOLOM, Montreal

4100 Sherbrooke Street West, Westmount, QC, H3Z 1A5

Tel: 514-937-3575 | http://www.templemontreal.ca

BETH TZEDEC REUBEN AND HELENE DENNIS MUSEUM, Toronto

1700 Bathurst Street, Toronto, ON, M5P 3K3

Curator: Dorion Liebgott

Tel: 416-781-3511 | museum@beth-tzedec.org | http://www.beth-tzedec.org/museum/

EDWARD BRONFMAN MUSEUM, CONGREGATION SHAAR HASHOMAYIM, Montreal

450 Kensington Avenue, Westmount, QC, H3Y 3A2

Curators: Elaine Hershenfield and Deanna Mendelson

Tel: 514-937-9471 | admin@theshaar.org | http://www.shaarhashomayim.org/museum

JEWISH ARCHIVES AND HISTORICAL SOCIETY OF EDMONTON AND

NORTHERN ALBERTA (JAHSENA), Edmonton

10220-156 St., Suite 200, Edmonton, AB, T5P 2R1

Archivist: Colleen Paull

Tel: 780-489-2809 | jahsena@shaw.ca | http://www.jahsena.ca/jahsena_001.htm

JEWISH GENERAL HOSPITAL ARCHIVES / ARCHIVES DE L'HOPITAL GÉNÉRAL JUIF, Montreal 3755 Chemin de la Côte Ste-Catherine Rd., A-200, Montreal, QC, H3T 1E2

Archivist: Linda Lei

Tel: 514-340-822, ext. 3277 | legacy@jgh.mcgill.ca | http://www.jgh.ca/en/archives

JEWISH HERITAGE CENTRE OF WESTERN CANADA, Winnipeg

C116 - 123 Doncaster Street, Winnipeg, MB, R3N 2B2

Archivist: Andrew Morrison

Tel: 204-477-7460 ext. 7461 | jhc@jhcwc.org | http://www.jhcwc.mb.ca/

JEWISH HISTORICAL SOCIETY OF SOUTHERN ALBERTA, Calgary

1607-90th Ave. S.W., Calgary, AB, T2V 4 V7

Archives Director: Roberta Kerr

Tel: 403-444-3171 | jhssa@shaw.ca | http://jhssa.org/

JEWISH MUSEUM AND ARCHIVES OF BC, Vancouver

6184 Ash Street, Vancouver, BC, V5Z 3G9

Archivist: Alysa Routtenberg

Tel: 604-638-7286 | Museum: 604-257-5199 | archives@jewishmuseum.ca | http://www.jewishmuseum.ca

\section{JEWISH PUBLIC LIBRARY ARCHIVES /}

ARCHIVES DE LA BIBLIOTĖQUE PUBLIQUE JUIVE, Montreal

1 carré Cummings Square, 5151 Côte Ste-Catherine Rd., Montreal, QC, H3W 1 M6

Archivist: Jessica Zimmerman | Archivist Emeritus: Eiran Harris

Tel: 514-345-2627, ext. 3015, 3000 | archives@jplmontreal.org | http://www.jewishpubliclibrary.org

LIBRARY AND ARCHIVES CANADA / BIBLIOTHĖQUE ET ARCHIVES CANADA, Ottawa

395 Wellington Street, Ottawa, ON, K1A 0N4

Archives search: http://www.collectionscanada.gc.ca/lac-bac/search/arch

Reference questions by phone: 1-866-578-7777 (toll-free in Canada and US)

Reference questions by email: http://www.bac-lac.gc.ca/eng/assistance-request-form 
MONTREAL HOLOCAUST MUSEUM ARCHIVES / ARCHIVES DU MUSÉE DE L'HOLOCAUSTE DE MONTRÉAL, Montreal

1 Carré Cummings Square, 5151, Côte Ste-Catherine Road, Montreal, QC, H3W 1 M6

Tel: 514-345-2605 | info@museeholocauste.ca | http://www.museeholocauste.ca

MUSEUM OF JEWISH MONTREAL / MUSÉE DU MONTRÉAL JUIF, Montreal 4040 St. Laurent \#R01, Montreal, QC, H2W 1 Y8

Director /Directeur: Zev Moses

Tel: 514-840-9300 | info@imjm.ca | http://mimj.ca

ONTARIO JEWISH ARCHIVES, Toronto

4600 Bathurst Street, Toronto, ON, M2R 3V2

Archives Director: Dara Solomon

Tel: 416-635-2883 ext. 187 | oja1@ujafed.org | www.ontariojewisharchives.org

OTTAWA JEWISH ARCHIVES, Ottawa

21 Nadolny Sachs Private, Ottawa, ON, K2A 1R9

Tel: 613-798-4696 ext. 260 | archives@jewishottawa.com | http://www.jewishottawa.org

SAINT JOHN JEWISH HISTORICAL MUSEUM ARCHIVES, Saint John

91 Leinster Street, Saint John, NB, E2L 1 J2

Curator/Archivist: Katherine Biggs-Craft

Tel: 506-633-1833 | sjjhm@nbnet.nb.ca|http://jewishmuseumsj.com 